\title{
Experimental evaluation of a coaxial needle insertion assistant with enhanced force feedback
}

\author{
Danilo De Lorenzo, Yoshihiko Koseki, Elena De Momi, \\ Kiyoyuki Chinzei Member, IEEE, and Allison M. Okamura Fellow, IEEE
}

\begin{abstract}
During needle insertion in soft tissue, detection of change in tissue properties is important both for diagnosis to detect pathological tissue and for prevention to avoid puncture of important structures. The presence of a membrane located deep inside the tissue results in a relatively small force variation at the needle tip that can be masked by relatively large friction force between the needle shaft and the surrounding tissue. Also, user perception of force can be limited due to the overall small force amplitude in some applications (e.g. brain surgery).

A novel robotic coaxial needle insertion assistant was developed to enhance operator force perception. The coaxial needle separates the cutting force at the needle tip from shear friction on the needle shaft. The assistant is force controlled (admittance control), providing the operator with force feedback that is a scaled version of the force applied by the needle tip to the tissue. The effectiveness of the assistant in enhancing the detection of different tissue types was tested experimentally. Users were asked to blindly insert a needle into artificial tissues with membranes at various depths under two force feedback conditions: (1) shaft and tip force together, and (2) only tip force. The ratio of successful to unsuccessful membrane detection was significantly higher when only the needle tip force is displayed to the user. The system proved to be compliant with the clinical applications requirements.
\end{abstract}

\section{INTRODUCTION}

D URING standard manual needle insertion procedures the operator manually inserts a straight needle inside the tissue in order to perform diagnosis (biopsy, blood sampling) or treatment (drug delivery, electrode placement) [1]. For several types of standard procedures (e.g. epidural anesthesia, brain biopsy, electrode placement for deep brain stimulation) the surgeon cannot see the path of the needle inside the tissue

This work was supported by National Institutes of Health under Grant R01 EB006435, Scuola Interpolitecnica di Dottorato Milano Torino e Bari, Japan Society for the Promotion of Science under Excellent Young Researcher Overseas Visit Program, Johns Hopkins University, Advanced Industrial Science and Technology, and Politecnico di Milano.

D. De Lorenzo is with Politecnico di Milano, Bioengineering Department, Neuroengineering and Medical Robotics Laboratory, Piazza Leonardo Da Vinci 32, 20133 Milano, Italy. (e-mail: danilo.delorenzo@mail.polimi.it)

Y. Koseki is with Research Institute of Human Life Technology, Advanced Industrial Science and Technology (AIST), Tsukuba, Japan.

E. De Momi is with Politecnico di Milano, Bioengineering Department, Neuroengineering and Medical Robotics Laboratory and with Istituto di Tecnologie Industriali ed Automazione, Consiglio Nazionale delle Ricerche, Milano, Italy.

K. Chinzei is with Research Institute of Human Life Technology, Advanced Industrial Science and Technology (AIST), Tsukuba, Japan.

A. M. Okamura is with the Department of Mechanical Engineering, and the Laboratory for Computational Sensing and Robotics, Johns Hopkins University, Baltimore, MD, USA

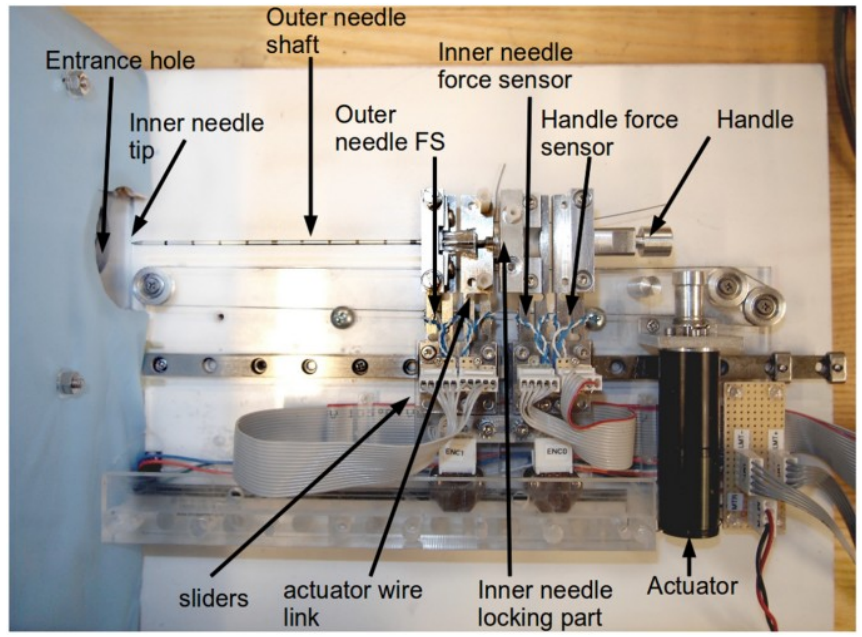

Fig. 1. Coaxial needle experimental setup. The blue sheet on the left is covering the tissue sample leaving only the entry point visible.

and thus must rely on his limited sense of touch or on a pre-planned path based on preoperative medical images. Enhancing the perception of the force at the needle/tissue interface could drastically increase the success of needle-based procedures [2]-[4]. The choice of which interaction force is displayed to the operator may have a significant impact on performance. The presence of a different type of tissue can be identified, for example, by a relatively small force variation at the needle tip (cutting force). Unfortunately, this small variation is easily masked by the relatively large friction force between the needle shaft and the surrounding tissue, which significantly complicates manual discrimination of the change in tissue property. Force feedback to enhance detection of small force variations is enabled by robotic devices. This can be achieved with two different types of robotic architectures: teleoperation, where the user is remotely maneuvering a device, or the cooperative manipulation, where the user and the robot cooperatively move the surgical instrument (needle). The control method of most of teleoperated devices is impedance control [5]-[7], while in cooperative manipulation, admittance control is preferred to allow force-to-motion scaling [8].

In previous work [9], we proposed an instrumented coaxial needle to measure cutting force separately from the shear friction. The exact force profile depends on several factors, including insertion speed, tissue properties, and friction. Here, we propose a robotic coaxial needle insertion assistant with a 
cooperative manipulation architecture, where the robot and the surgeon simultaneously drive a surgical coaxial needle. We consider how to display only the cutting force to an operator during needle insertion in order to improve operator perception.

Our purpose was to measure the effectiveness of the system in helping the operator detect membranes inside tissues. This is useful, for example, in keyhole neurosurgical interventions, where the possibility to detect unexpected situations, like touching a vessel, could lead to vessel rupture (puncture) and consequently to bleeding inside the brain parenchyma.

The performance of the system was tested in an experiment in which inexperienced users inserted needles into artificial tissues, and attempted detect the presence of a membrane under the influence of two different control algorithms. In the first algorithm, both the cutting and the friction force were fed back to the user; in the second, only the cutting force at the needle tip was presented.

\section{Materials AND Methods}

Our cooperative manipulation device is equipped with a standard coaxial needle. The outer needle covers the side of the inner needle, in order to prevent the friction between the needle and surrounding tissue from being applied to the inner needle. The tip of the inner needle is not covered, so that the cutting force can be applied to the inner needle. Both the inner and the outer needles are inserted by the operator with the help of an actuator, so that the operator can feel an amplified version of the force on the needle.

\section{A. Coaxial Needle Insertion Assistant Design}

Fig. 1 shows the coaxial needle insertion assistant. The two parts of the coaxial needle were mounted on separate sliders and moved along a linear guide. The inner needle slider was equipped with a handle for the operator to push it. The needle slider was driven via a transmission cable by a geared DC motor. A plate joined the inner and outer needle sliders. The system has one-degree-of-freedom linear motion along the needle axis $(10 \mathrm{~mm} / \mathrm{sec}$ maximum velocity, $10 \mathrm{~N}$ maximum force output, and $130 \mathrm{~mm}$ stroke). It is equipped with four force sensors able to sense the inner needle, outer needle, operator, and motor forces. The inner needle force sensor was designed to measure $10 \mathrm{~N}$ maximum, and others were designed to measure $40 \mathrm{~N}$ maximum, since the cutting force is smaller than the other forces. Each force sensor was a flexure parallelogram mechanism in Wheatstone bridge configuration. The bridge output was amplified, and the force sensors were calibrated with weights. The needle position was measured by an optical linear encoder. The force signals, the position signals, and the control algorithm were acquired and refreshed at $5 \mathrm{kHz}$ by means of a real-time operating system.

\section{B. Coaxial Needle Insertion Assistant Control}

Fig. 2 shows the scheme of the force-controlled needle

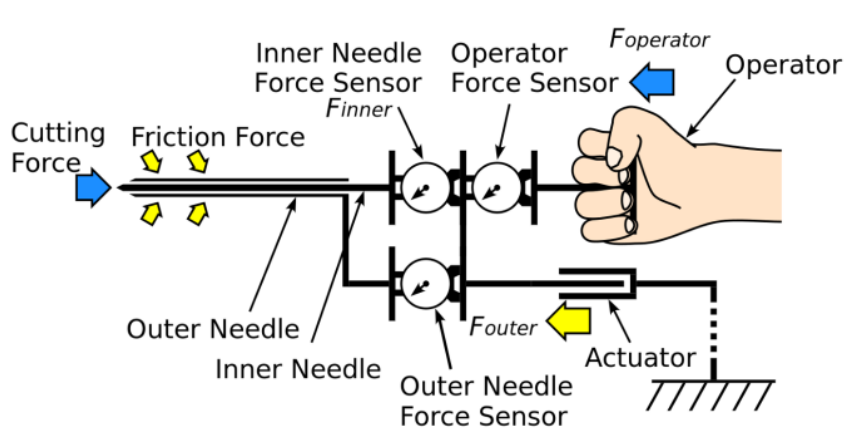

Fig. 2. Overview of coaxial needle insertion assistant. Three force sensors are used to sense the total force applied by the operator, the force between the outer needle and the tissue (friction force) and the force applied by the inner needle to the tissue (at the tip). An actuator controls the motion of the outer and inner needle together such that the operator receives force feedback that is a scaled version of the force applied to the tissue.

insertion assistant. The tip of the inner needle cuts the tissue and the outer needle covers the inner needle, preventing the shear friction between the needle and the surrounding tissue from acting on the inner needle (Fig. 2). The configuration allows a force sensor placed on the opposite side of the tip of the inner needle to detect the cutting force.

The force control loop allows the operator force $\left(F_{\text {operator }}\right)$ to be proportional to the cutting force measured by the inner needle $\left(F_{\text {operator }}=\alpha F_{\text {inner }}\right)$, so that the force-controlled actuator can amplify the forces between the needle and the tissue and present it to the operator. An outer loop implicit force trajectory-tracking controller [9] was implemented to determine the desired needle position $\left(\mathrm{x}_{d}\right)$ :

$$
x_{d}(t)=k_{i f} \int_{0}^{t} \Delta_{f}(t) d t+k_{p f} \Delta_{f}
$$

where $\Delta f=F_{\text {operator }}-\alpha F_{\text {inner }}$ and $k_{i f}$ and $k_{p f}$ are the proportional and integral outer loop control gains [9]. The inner loop position-control is a PID controller. The PID is implemented to set the actuator output $\left(F_{a c t}\right)$ :

$$
F_{\text {act }}=k_{p} P_{e}+k_{i} \int P_{e}+k_{d} \dot{P}_{e}
$$

where $P_{e}$ is the position error between the actual and desired robot positions.

In this type of control, the position of the robot is controlled to minimize the error between the measured and desired tip forces, so that the user can feel an amplified version of the needle/tissue interaction forces.

\section{Experiment Design}

The goal of the experiment was to compare user performance of membrane detection prior to puncture with two types of force feedback to the user: an amplified version of the cutting force alone and an amplified version of the cutting plus the friction force during a needle insertion task. 


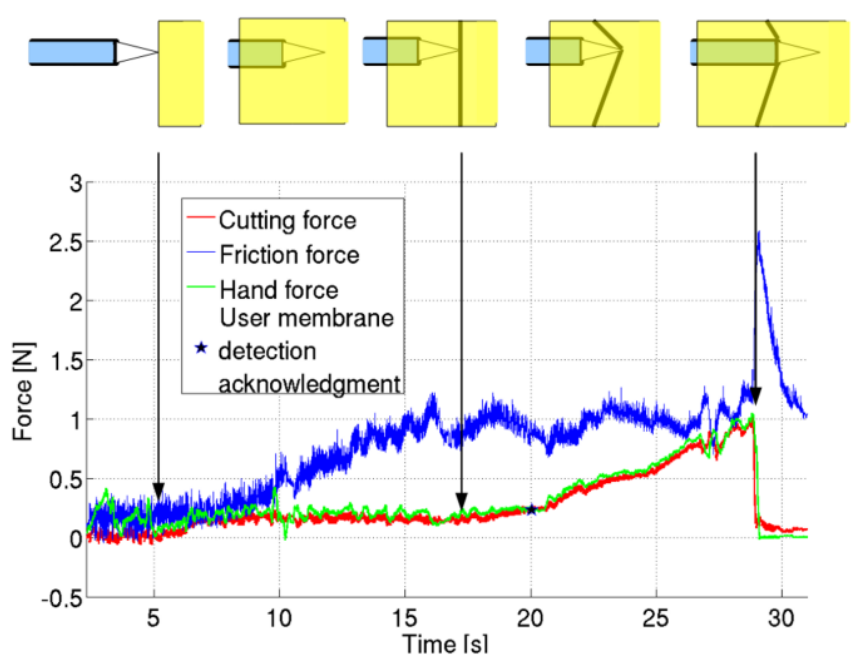

Fig. 3. Example of signals for a successful insertion where the user (star) detect the presence of the membrane before the puncture. The graph shows all the phases of the needle insertion: free motion in air, surrounding tissue entering, membrane touching and puncture.

The latter represents what the operator feels inserting the needle in a standard manual procedure, short of the amplification gain.

Users were asked to blindly insert a needle into artificial tissue and to press a button as soon as they perceived a membrane. The time at which the button press occurred was recorded, together with position and force signals. Then the users were asked to keep inserting the needle until they perceived the membrane puncturing, and to stop advancing the needle immediately after puncture. The insertion was considered a "success" only if the user pressed the button within a time window that starts at the time the needle touches the membrane and ends immediately before the puncture occurs (Fig. 3).

Fig. 1 shows the experimental setup. Six samples of PVC rubber to mimic the soft tissue were prepared. Two membranes made of a harder silicone material (Smooth-on, 910 ) with 0.4 and $0.8 \mathrm{~mm}$ thickness were placed inside the soft artificial tissue at three different depths from the outer surface: 15,35 , and $55 \mathrm{~mm}$. The samples were fixed in order to prevent unwanted relative motion between the tissue and base of the robot. A steel coaxial needle designed for biopsy (C1616B, Bard: $15 \mathrm{G}$ ) with $2 \mathrm{~mm}$ diameter was used.

Eleven right-handed, neurologically healthy, non-medical-professional users participated in this study. The study was approved by the Johns Hopkins University Homewood Institutional Review Board. The users were seated holding the handle of the needle with the right hand. The tissue was covered except for the entry point of the needles, so that the users could not visually discern the position of the membrane inside the samples. Each user randomly performed one needle insertion under all combinations of the two force feedback types (cutting force, cutting + friction force), two membrane thicknesses, and three membrane depths $(15,35$, and $55 \mathrm{~mm})$. The force feedback

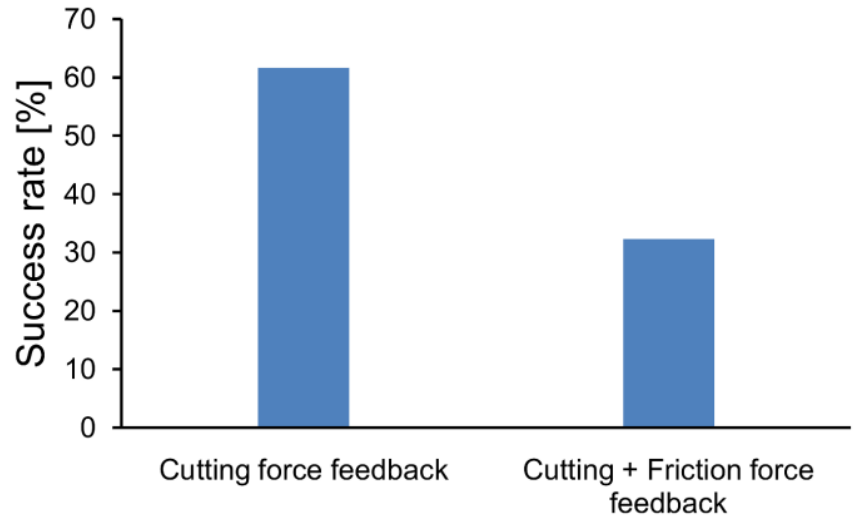

Fig. 4. Rate of success in membrane detection for the two types of force feedback to the user.

amplification gain was set to $\alpha=6$. The value was chosen in order to guarantee that the amplified force feedback given to the user was well above the noisily friction forces between the sliders and the linear guide that could mask the small tissue cutting force $(\sim 1 \mathrm{~N} \max )$ in case of low amplification. The stability of the system and the maximum force exerted by the motor was the upper boundary for the gain value. The total number of insertions performed by each user was 12 and the total number of insertions performed by all users was 132 . The insertion order for all these parameters was randomly changed among users. Each user performed 15 minutes of practice to understand the device behavior. The conditions of the practice sessions were also sequenced pseudo-randomly.

\section{RESULTS}

Fig. 3 shows the cutting force, friction force and hand force (divided by the force gain, for comparison) profiles. The cutting force curve shows an almost constant force inside the artificial tissue (from 7 to 17 seconds), then a force increment at the time of membrane touching (at 17 seconds) and the typical quick force drop after membrane puncturing (at 28 seconds). The friction force gradually increases until the tip of the needle reaches the membrane. After that point, the cutting force is rapidly increasing and the user perceives the force quickly growing. During that time window, the friction force remains almost constant, due to the very short needle travel. Immediately after the membrane puncture, the friction force rapidly increases because the membrane meets the outer needle and starts pushing it. After the user stops, the hand and cutting force goes to zero, while the friction force relaxes to the value prior to the membrane touch. In Fig. 4, the success rate is shown for the two types of control. The other conditions (membrane thickness, gain amplification, and membrane depth) are summed for each controller type. With only the cutting force displayed, the operator could detect the presence of a membrane before its rupture in around the $62 \%$ of all the insertions, in comparison to $32 \%$ when the friction force is added.

Fig. 5 shows the success rate in membrane detection with 


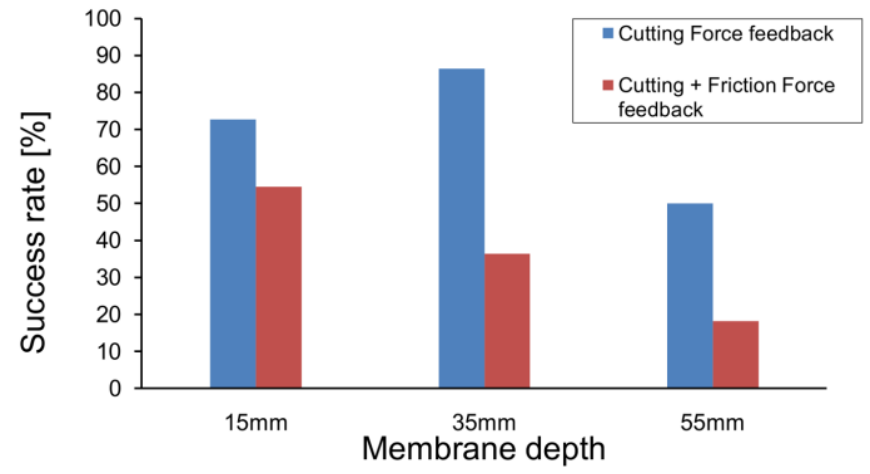

Fig. 5 Success rate in membrane detection for the two types of force feedback to the user, with respect to the membrane position (depth) inside the artificial soft tissue sample.

respect to membrane depth and control type. Again, the success rate is always greater when only the cutting force is displayed to the user, in comparison to when cutting and friction force are displayed together $(72 \%$ vs. $54 \%$ for a 15 mm membrane depth, $86 \%$ vs. $36 \%$ for a $35 \mathrm{~mm}$ depth, and $50 \%$ vs. $18 \%$ for a $55 \mathrm{~mm}$ depth). For the friction and cutting force control, performance linearly decreases with the increased membrane depth.

\section{DISCUSSION AND CONCLUSION}

It was experimentally shown that the coaxial needle assistant facilitated perception of thin membranes during needle insertion when an amplified version of the cutting force at the needle tip is displayed to the user. Results showed that this control method performs better than displaying the overall interaction forces between the needle and the tissue (cutting and friction forces together). This intuitively follows from Weber's Law, which states that the ratio of the increment threshold to the background intensity is a constant. In case of large background load, small variations are not felt by the human operator, specially if the operator is inserting the needle without the help of the robot assistant. As expected, the gap in performance increases with membrane depth inside the surrounding tissue; the larger the depth, the larger the friction force that masks the small force variations due to cutting at the needle tip.

Further studies could consider the coaxial needle insertion device as a training tool. Previous studies showed that the role of haptic feedback could be different for experienced and inexperienced surgeons [2][10][11]. Our results are promising in terms of clinical practice, since enhanced perception performs well in a variety of different conditions.

With a conventional needle, soft membranes are generally not detectable due to friction. Even with coaxial needles, if the cutting force is not amplified, membrane detection is hard due to the overall small amplitude forces detected in particular clinical applications (e.g. brain surgery).

The device is able to sense the cutting force at the needle tip and the friction force along the needle without sensors placed directly on the needle. This avoids sterilization and miniaturization problems and allows standard needle usage. However, friction between the inner and outer needles degraded the haptic feedback to the user, especially for high force gain, since the user felt not only the cutting force at the tip of the needle, but also the friction force from the outer needle moving relative to the inner needle. In case of large friction force between the inner and the outer needle, the system also exhibited large oscillations. Even if needle cleaning solves the problem, the role of needle-needle friction should be further investigated with regard to the control algorithm. Also, friction is strictly correlated with misalignment between the outer needle and the inner one. Improvements to the apparatus design could improve alignment.

The role of the force feedback amplification needs to be investigated to understand how the gain can improve or degrade the force friction cancellation and the membrane detection performances. The system should be carefully tested in preclinical conditions, such as ex vivo biological tissues, to better evaluate clinical relevance.

The ability of our system to display to the user only the tip force could help the operator in all the procedures that involve needle insertion, where the detection of membranes or different type of tissue can improve the performance or the success of the intervention.

\section{REFERENCES}

[1] N. Abolhassani, R. Patel, and M. Moallem, "Needle insertion into soft tissue: A survey," Medical Engineering and Physics, vol. 29, pp. 413-431, 2007.

[2] C. R. Wagner and R. D. Howe, "Force feedback benefit depends on experience in multiple degree of freedom robotic surgery task," IEEE Transactions on Robotics, vol. 23, pp. 1235-1240, 2007.

[3] T. Ortmaier, et al., "Robot assisted force feedback surgery," in Advances in Telerobotics, Springer Tracts in Advanced Robotics, vol. 31, pp. 361-379, 2007.

[4] M. Mahvash, et al., "Force-feedback surgical teleoperator: Controller design and palpation experiments," in Proc. Symposium on Haptic Interfaces for Virtual Environment and Teleoperator Systems, pp. 465-471, 2008.

[5] M. Tavakoli, R. V. Patel, and M. Moallem, "Bilateral control of a teleoperator for soft tissue palpation: Design and experiments," in Proc. IEEE International Conference on Robotics and Automation, pp. 3280-3285, 2006.

[6] L. H. Eadie, A. M. Seifalian and B. R. Davidson, "Telemedicine in surgery," British Journal of Surgery, vol. 90, pp. 647-658, 2003.

[7] G. Hager, et al., "Surgical and interventional robotics: Part III," IEEE Robotics and Automation Magazine, vol. 15, pp. 84-93, 2008.

[8] J. Roy, D. L. Rothbaum, and L. L. Whitcomb, "Haptic feedback augmentation through position based adaptive force scaling: Theory and experiment," in Proc. IEEE/RSJ International Conference on Intelligent Robots and Systems, pp. 2911-2919, 2002.

[9] T. Washio and K. Chinzei, "Needle force sensor, robust, sensitive detection of the instant of needle puncture," in Proc. International Conference on Medical Image Computing and Computer-Assisted Intervention, pp. 113-120, 2004.

[10] O. Gerovichev, P. Marayong and A. M. Okamura, "The effect of visual and haptic feedback on manual and teleoperated needle insertion," in Proc. Fifth International Conference on Medical Image Computing and Computer Assisted Intervention, pp. 147-154, 2002.

[11] D. L. Rothbaum, et al., "Task performance in stapedotomy: Comparison between surgeons of different experience levels," Otolaryngology - Head and Neck Surgery, vol. 128, pp. 71-77, 2003. 